\title{
Ureterostomía cutánea como derivación urinaria definitiva en trasplante renal
}

\section{Cutaneous ureterostomy as definitive urinary diversion in kidney transplant}

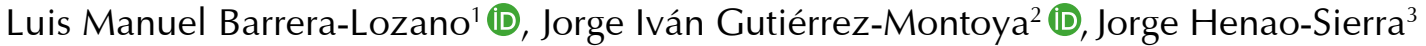

\begin{abstract}
MD, FACS, cirujano de Trasplantes de Órganos Abdominales, Unidad Funcional de Trasplantes y Enfermedades Digestivas, Servicio de Cirugía HPB/Trasplantes de Órganos Abdominales/Rehabilitación Intestinal, Hospital San Vicente Fundación, Rionegro, Colombia 2 Médico, cirujano de Trasplantes de Órganos Abdominales, Unidad Funcional de Trasplantes y Enfermedades Digestivas, Servicio de Cirugía HPB/Trasplantes, Hospital Universitario San Vicente Fundación, Universidad de Antioquia, Medellín, Colombia

3 Médico especialista en Medicina interna y Nefrología, Nefrón S.A., Universidad de Antioquia, Medellín, Colombia
\end{abstract}

\section{Resumen}

Introducción. Cerca del 15\% de los pacientes con insuficiencia renal crónica terminal tienen alteraciones de las vías urinarias inferiores. Estas anomalías eran consideradas una contraindicación para el trasplante renal. Por lo anterior, el objetivo del presente trabajo es describir el comportamiento sociodemográfico y clínico de los pacientes trasplantados renales con ureterostomía cutánea como técnica de derivación definitiva de las vías urinarias.

Métodos. Se realizó un estudio descriptivo, longitudinal y retrospectivo de los pacientes trasplantados renales con vejiga anormal y ureterostomía cutánea, entre enero de 1973 y octubre de 2012.

Resultados. En 4.294 trasplantes renales, se practicaron 24 (0,55\%) ureterostomías, I9 (79,I \%) como técnica inicial y 5 por falla de la ureteroneocistostomía. Diez ( $4 \mathrm{I}, 7 \%)$ ureterostomías fallaron, la mayoría ( 8 casos) por estenosis.

Se presentó infección urinaria en 20 pacientes y la mortalidad fue del 8,3\% (2/24). El 50 \% (I2/24) de los pacientes con trasplante de riñón estuvieron libres de infección urinaria durante el primer año. La supervivencia del riñón trasplantado fue de 93,8 \% (23/24) a los I8 meses, de 85,9\% (20/24) a los 36 meses y de 66,7\% (I6/24) a los 50 meses de seguimiento. La supervivencia de los injertos con ureterostomía sin infección fue del Ioo \% durante el periodo de seguimiento, mientras que la supervivencia de los riñones con infección urinaria fue de $93 \%(23 / 24)$ a los I8 meses, de $76 \%$ (I8/24) a los 36 meses y de $54 \%(13 / 24)$ a los 50 meses ( $\mathrm{p}=0,235)$.

Conclusiones. La ureterostomía cutánea es una alternativa segura para la derivación urinaria en pacientes trasplantados renales con alteraciones de la vejiga que no permite su uso o preparación antes del trasplante.

Palabras clave: riñón; trasplante de riñón; vejiga urinaria; uréter; ureterostomía.

Fecha de recibido: 3/10/2019 - Fecha de aceptación: 17/01/2020

Correspondencia: Luis Manuel Barrera-Lozano, Hospital San Vicente Fundación Rionegro, Rionegro, Colombia.

Teléfono: (574) 448-2626, extensión 3423

Correo electrónico: luismanuelbarrera@gmail.com y luis.barrera@sanvicentefundacion.com

Citar como: Barrera-Lozano LM, Gutiérrez-Montoya JI, Henao-Sierra J. Ureterostomía cutánea como derivación urinaria definitiva en trasplante renal. Rev Colomb Cir. 2020;35:630-8. https://doi.org/10.30944/20117582.485

Este es un artículo de acceso abierto bajo una Licencia Creative Commons - BY-NC-ND https://creativecommons.org/licenses/by-ncnd/4.0/deed.es 


\begin{abstract}
Introduction. About $15 \%$ of patients with end-stage chronic renal failure have lower urinary tract abnormalities. These abnormalities were considered a contraindication for kidney transplantation. Therefore, the objective of the present work is to describe the sociodemographic and clinical behavior of renal transplant patients with skin ureterostomy as a definitive urinary tract bypass technique.
\end{abstract}

Methods. A descriptive, longitudinal and retrospective study of renal transplant patients with abnormal bladder and skin ureterostomy was conducted between January 1973 and October 2012.

Results. We performed 24 (0.55\%) ureterostomies in 4.294 kidney transplants. Nineteen (79.I\%) were used as first and definitive urinary diversion, and five were ureteroneocystostomies that failed and required ureterostomy as alternative diversion. Ten (4I.7\%) ureterostomies failed, most (eight cases) from stenosis. Urinary tract infection (UTI) were present in 20 patients and mortality was 8.3\% (2/24); 50\% (I2/24) of kidney transplants were free of urinary tract infection during the first year. The survival of the transplanted kidney was $93.8 \%(23 / 24)$ at 18 months, $85.9 \%(20 / 24)$ at 36 months, and $66.8 \%(16 / 24)$ at 50 months of follow up. The survival of grafts with ureterostomy without urinary tract infection were $100 \%$ during the follow-up period, while the survival of the kidneys with UTI was $93 \%$ (23/24) at I 8 months, $76 \%$ (I8/24) at 36 months, and 54\% $(\mathrm{I} 3 / 24)$ at 50 months $(\mathrm{p}=0.235)$.

Conclusions. Cutaneous ureterostomy is a safe alternative for urinary diversion in transplanted renal patients with bladder abnormalities that cannot be prepared or used for a routine diversion before transplantation.

Keywords: kidney; kidney transplantation; urinary bladder; ureter; ureterostomy.

\section{Introducción}

Cerca del $15 \%$ de los pacientes con insuficiencia renal crónica terminal tienen alteraciones de las vías urinarias inferiores ${ }^{\mathrm{I}}$. Entre el 20 y el $30 \%$ de los trasplantes renales en la población pediátrica son causados por anormalidades de las vías urinarias inferiores ${ }^{2}$. Varios reportes han demostrado que la disfunción vesical afectaría negativamente la función del injerto ${ }^{3}$.

La vejiga puede ser anormal por varias alteraciones de las vías urinarias inferiores, como la neuropatía que produce vejiga neurogénica, la obstrucción estructural en la salida de la vejiga (estenosis uretral, válvulas uretrales posteriores), el vaciado disfuncional adquirido, las cicatrices perivesicales, el aumento del tamaño vesical y las enfermedades vesicales adquiridas, entre las que se encuentran la cistitis intersticial, la fibrosis, los cambios posteriores a la radioterapia y las infecciones. Estas condiciones hacen que la vejiga permanezca contraída, lo cual reduce su capacidad de llenado o impiden que pueda vaciarse ${ }^{4-6}$.
Las anomalías de las vías urinarias inferiores que conllevan una vejiga anormal eran consideradas una contraindicación para el trasplante renal, pero ahora se considera que, con una buena evaluación de la vía urinaria antes del trasplante y eligiendo el procedimiento apropiado para su corrección, se pueden lograr supervivencias del injerto y del paciente similares a las obtenidas cuando no existen tales anomalías ${ }^{4}$.

En el trasplante renal, se prefiere hacer el implante en la vejiga del paciente receptor cuando su función y capacidad son adecuadas, pero cuando no lo es, se tienen varias opciones de intervención, entre las que se encuentran el aumento del tamaño vesical, el drenaje por los conductos ileales, la derivación urinaria continente, la cateterización intermitente de la vejiga, el drenaje vesical mediante un conducto de tipo Mitrofanoff y la ureterostomía cutánea ${ }^{5}$. Las anteriores opciones presentan como principal problema el aumento de las infecciones urinarias (56\% de los pacientes con aumento del tamaño 
vesical) ${ }^{7}$, las cuales tienen un impacto directo en la supervivencia del injerto ${ }^{8}$.

La ureterostomía cutánea, una alternativa propuesta por Amín en 1971, es técnicamente más sencilla, no conlleva alteraciones metabólicas por el uso del intestino como en las otras técnicas de derivación y ha presentado en su serie de pacientes tasas de infección urinaria que alcanzan el 42,8 \% ${ }^{9-17}$. Sin embargo, hasta el momento, no hay 'evidencia' contundente que favorezca una única alternativa de derivación sobre las otras en pacientes con vejigas anormales que vayan a ser sometidos a trasplante renal.

Por lo anterior, el objetivo del presente trabajo es describir el comportamiento sociodemográfico y clínico de los pacientes con trasplantes renales y ureterostomía cutánea como técnica de derivación definitiva de las vías urinarias.

\section{Métodos}

Se llevó a cabo un estudio observacional, longitudinal y retrospectivo de una serie de pacientes con trasplantes renales de donante fallecido o vivo, relacionado con vejiga anormal, a quienes se le practicó ureterostomía primaria definitiva como técnica de derivación urinaria, o pacientes que requirieron cambio de su ureteroneocistostomía a ureterostomía como técnica alternativa por fallo de la anastomosis a la vejiga.

Se excluyeron aquellos pacientes que presentaron necrosis ureteral en el periodo posoperatorio temprano (primeras 24 horas después del trasplante) que impidió la continuidad o la realización de la ureterostomía cutánea, por pérdida del injerto debido a complicaciones vasculares o inmunológicas hiperagudas.

Dichos pacientes corresponden a los trasplantados del Programa de Trasplante de Órganos Abdominales del Hospital Universitario San Vicente Fundación, centro de IV nivel de atención de Medellín, Colombia, desde 1973 hasta el 2012.

Las variables se clasificaron como: demográficas, etiológicas, quirúrgicas, terapia inmunosupresora, complicaciones de la derivación, inmunosupresión y resultados.
Los resultados evaluados fueron: infección urinaria comprobada mediante urocultivo positivo con Ioo.ooo UFC más signos de reacción inflamatoria sistémica, disfunción del injerto evidenciado por aumento súbito de la creatinina basal, o infección y disfunción concomitantes. Otro fue la falla de la ureterostomía cutánea, definida como cualquier complicación asociada o inherente a la técnica de derivación urinaria y que generara cambios súbitos o abruptos de la creatinina basal, entre las cuales se encuentran la estenosis de la ureterostomía, la necrosis, el sangrado y otros no anticipados asociados con el procedimiento. También se evaluó la supervivencia de los injertos renales, que obvia la necesidad definitiva de terapias de reemplazo renal, y la mortalidad.

El grupo de estudio se evaluó mediante un formulario con preguntas sobre cada una de las variables y resultados considerados, relacionados con la persona y las fases de tratamiento médico o quirúrgico que fueron llevadas a cabo durante su hospitalización y en el control por consulta externa de los servicios de Nefrología o de Cirugía de Trasplantes. Las fuentes de información fueron el protocolo anterior al trasplante, la historia clínica durante la hospitalización por el trasplante renal y los posteriores reingresos por complicaciones asociadas y, además, las anotaciones de los seguimientos por consulta externa en la Unidad de Trasplantes del Hospital Universitario San Vicente Fundación. Aquellos datos no consignados en la historia clínica se obtuvieron mediante llamada telefónica directa al paciente. Los datos obtenidos se recopilaron en una base de datos diseñada en Microsoft Access $2007^{\mathrm{TM}}$.

Por ser un estudio observacional, retrospectivo y sin ninguna intervención, se utilizó el consentimiento informado del paciente al momento de su ingreso a la institución para ser trasplantado, ya que este no solo autoriza a los cirujanos y a la institución a practicar el procedimiento, sino también a usar los datos para investigación. 


\section{Análisis estadístico}

El análisis incluyó una fase descriptiva y otra analítica. En la fase descriptiva, se estudiaron las variables cualitativas y cuantitativas, se presentaron sus frecuencias, promedios, desviaciones estándar, medianas y rangos. En la fase analítica, se evaluaron los tiempos libres de infección urinaria, la supervivencia de los injertos y de los pacientes mediante el método de Kaplan-Meier, y se comparó la supervivencia de los injertos en pacientes con infecciones urinarias con la de aquellos que no la presentaron, con la prueba de log rank.

\section{Resultados}

\section{Características demográficas y clínicas iniciales}

De 4.294 trasplantes renales practicados durante los 39 años que lleva el programa (1973-2012), en $24(0,55 \%)$ se practicó ureterostomía como técnica de derivación urinaria, a quienes se les hizo seguimiento de 60,5 meses en promedio; 23 (93,8 $\%)$ correspondieron a donante fallecido y uno a donante vivo relacionado. De las ureterostomías, $20(83,3 \%)$ se practicaron en el primer trasplante y cuatro (I6,7 \%) en el segundo. El 79,2 \% (I9/24) de los pacientes sometidos a ureterostomía fueron de sexo masculino, la edad promedio fue de 24,2 años (rango de 3 a 50 años) y I2 (50 \%) eran menores de 18 años al momento del trasplante.

En 6/24 (25\%) de las ureterostomías, la principal causa de falla renal crónica terminal fue la uropatía obstructiva por valvas uretrales posteriores. Le siguieron el reflujo vésico-ureteral, las infecciones urinarias a repetición y la glomerulonefritis no especificada (tabla I).

Solo dos de los 24 trasplantados renales no tenían ninguna alteración vesical. Los otros 22 pacientes presentaron alteraciones que se relacionan en la tabla 2 e indicaron la ureterostomía como alternativa de derivación urinaria, por alteraciones uretrales o notable disminución de la capacidad vesical (secundaria a tuberculosis).

Para la inducción, se utilizó alemtuzumab en 8 de los 24 casos, seguido por basiliximab en seis, timoglobulina en seis y solo metilprednisolona en cinco. El tiempo medio de isquemia fría fue de I8 horas con una desviación estándar de 6 horas y un rango de 25 minutos a 26 horas. El tratamiento inmunosupresor de mantenimiento más utilizado fue el triple (inhibidor de calcineurina más micofenolato mofetilo más esteroide), en once $(45,8 \%)$ de los 24 casos con tacrolimus y en otros once con ciclosporina. En 17 de los 24 riñones trasplantados se obtuvo excelente función del injerto, dos de los injertos tuvieron función lenta y cinco requirieron hemodiálisis en la primera semana postrasplante.

Tabla 1. Causas de falla renal crónica terminal

\begin{tabular}{lcc}
\hline \multicolumn{1}{c}{ Causa } & Frecuencia & $\%$ \\
\hline Diabetes mellitus & 1 & 4,2 \\
Hipertensión arterial sistémica & 1 & 4,2 \\
Vejiga neurogénica & 2 & 8,3 \\
Valvas uretrales posteriores & 6 & 25,0 \\
Síndrome de abdomen en pasa & 2 & 8,3 \\
Cloaca & 1 & 4,2 \\
Otros & 11 & 45,8 \\
\hline Total & 24 & 100,0 \\
\hline
\end{tabular}

Tabla 2. Causas de vejiga anormal

\begin{tabular}{lcc}
\hline \multicolumn{1}{c}{ Causa } & Frecuencia & $\%$ \\
\hline Valvas uretrales posteriores & 6 & 25,0 \\
Vejiga neurogénica & 4 & 16,7 \\
$\begin{array}{l}\text { Tuberculosis de vejiga, bajo } \\
\text { volumen }\end{array}$ & 3 & 12,5 \\
Síndrome de abdomen en pasa & 2 & 8,3 \\
Mielomeningocele & 2 & 8,3 \\
Estenosis uretral por trauma & 2 & 8,3 \\
pélvico & 1 & 4,2 \\
Estenosis uretral por duplicación & 1 & \\
uretral & 1 & 4,2 \\
Estenosis uretral posterior a & 1 & 4,2 \\
resección transuretral de próstata & 1 & 4,2 \\
Estenosis uretral & 24 & 4,2 \\
Mala definición sexual, epispadias & 24 \\
Cloaca & & 100 \\
Ninguna & & \\
\hline Total & & \\
\hline
\end{tabular}




\section{Características de las ureterostomías}

Se practicaron 19 ureterostomías cutáneas como técnica inicial de derivación de las vías urinarias durante el trasplante renal. En los otros cinco pacientes, se procedió inicialmente a la ureteroneocistostomía; en uno de ellos se había practicado aumento del tamaño vesical y drenaje vesical a través de un conducto de tipo Mitrofanoff antes del trasplante. Estas cinco derivaciones terminaron en ureterostomía cutánea como técnica de salvamento de las vías urinarias y del riñón trasplantado, por los siguientes motivos: tres casos de estenosis de la ureteroneocistostomía, un caso de filtración de la anastomosis y un caso de cistectomía radical por cáncer vejiga, la cual se practicó cinco años después del trasplante renal de donante vivo relacionado. Se practicó ureterostomía cutánea terminal en 18 de los 24 casos y técnica de asa lateral (Amin-Lich), en seis.

\section{Complicaciones de los injertos renales}

Diez (4I,7 \%) de las 24 ureterostomías fallaron por los siguientes motivos: estenosis de la ureterostomía en 8 casos, sangrado más estenosis en uno y necrosis de la ureterostomía en otro. Los fallos se manejaron con las siguientes alternativas terapéuticas: dilataciones periódicas en cuatro, reimplantación de la ureterostomía en tres, reconstrucción con el apéndice cecal en uno, reimplantación más nefrostomía percutánea en otro y solo un caso requirió reimplantación, reconstrucción y nefrostomía percutánea (tabla 3).

Tabla 3. Manejo de fallo de ureterostomía cutánea

\begin{tabular}{lcc}
\hline \multicolumn{1}{c}{ Tipo de manejo } & Frecuencia & $\%$ \\
\hline Sin fallo de ureterostomía & 14 & 58,3 \\
Dilataciones periódicas & 4 & 16,7 \\
$\begin{array}{l}\text { Reconstrucción con el apéndice cecal } \\
\text { (Mitrofanoff) }\end{array}$ & 1 & 4,2 \\
$\begin{array}{l}\text { Reimplantación del uréter } \\
\text { Reimplantación del uréter, nefrostomía }\end{array}$ & 3 & 12,5 \\
percutánea permanente & & 4,2 \\
$\begin{array}{l}\text { Reimplantación del uréter, } \\
\text { reconstrucción con el apéndice cecal } \\
\text { (Mitrofanoff), nefrostomía percutánea }\end{array}$ & 1 & 4,2 \\
permanente & & \\
\hline Total & 24 & 100,0 \\
\hline
\end{tabular}

En $9(37,5 \%)$ de los 24 pacientes con trasplante renal se presentó un episodio de rechazo durante el primer año, en 8 de ellos por rechazo celular agudo y en uno por rechazo mediado por anticuerpos.

Se presentó, al menos, un episodio de infección urinaria en el 83,3\% (20 casos) de los riñones trasplantados. En I2 de los 24 casos la infección se presentó en el primer año del procedimiento, 3 casos en el segundo, dos más en el tercer año y el mismo número en el cuarto año.

Cinco $(20,8 \%)$ de los 24 pacientes con trasplante renal entraron definitivamente a diálisis por pérdida del injerto, por las siguientes causas: pérdida inmunológica por rechazo celular agudo grave que no respondió a tratamiento en dos casos, pérdida de las vías urinarias en uno, pérdida del injerto por nefropatía crónica en otro, y choque hipovolémico que terminó en urgencia dialítica en otro paciente que, posteriormente, falleció durante la hospitalización.

Hubo dos $(8,3 \%)$ muertes entre los 24 pacientes con trasplante renal y ureterostomía cutánea. Uno de ellos falleció por choque séptico después de ruptura y filtración de la ureteroneocistostomía manejadas con ureterostomía; además, presentó función retardada del injerto, con peritonitis terciaria por Candida albicans en el cultivo de líquido peritoneal y bacilos Gram negativos en los hemocultivos; se le practicaron múltiples lavados de la cavidad y, finalmente, no mejoró con el tratamiento antibiótico de amplio espectro. El segundo paciente presentó trombosis venosa profunda de la vena innominada, las venas subclavias y las axilares, que se manejó con trombólisis por vía endovascular; sin embargo, presentó coagulación intravascular diseminada, choque hipovolémico y, finalmente, falleció.

\section{Tiempo libre de enfermedad, supervivencia de los injertos y de los pacientes}

La media de tiempo de seguimiento sin infecciones urinarias de los pacientes con ureterostomía, fue de 18,8 meses, con un rango intercuartílico (RIC) de 5,3 a 32,2 meses y una mediana de tres 
meses. El análisis muestra que i6 (66\%) de los 24 pacientes con ureterostomía estuvieron libres de infección en el primer mes después del trasplante, I2 $(48,9 \%)$ hasta el tercer mes, y solamente tres (I3\%) hasta los 32 meses de seguimiento (figura I).

La media de supervivencia del injerto renal en pacientes con derivación urinaria mediante ureterostomía cutánea, fue de I08,i meses ( $\mathrm{RIC}=80,5-\mathrm{I} 35,7)$. La supervivencia del riñón trasplantado fue de $93,8 \%$ a los I8 meses, de $85,9 \%$ a los 35 meses y de $66,8 \%$ a los 50 meses de seguimiento (figura 2).

La media de supervivencia de los pacientes fue de I30 meses (RIC=II2,9-I47,2); la supervivencia fue de $95,8 \%$ al mes de seguimiento, de 90,2 \% a los I9 meses y, posteriormente, se mantuvo constante (figura 3).

Posteriormente, se comparó la supervivencia de los injertos renales que se habían infectado en cualquier momento del seguimiento, con la de los que nunca sufrieron infecciones (figura 4). Se encontró que la supervivencia de los injertos renales en pacientes con ureterostomía y sin infecciones, fue del Ioo \% durante el periodo de seguimiento, mientras que la supervivencia de los riñones con infección urinaria fue de $93 \%(23 / 24)$ a los 18 meses, de $76 \%$ (I8) a los 36 meses y de $54 \%$ (I3) a los 50 meses. No obstante, lo anterior no fue estadísticamente significativo ( $\mathrm{p}=\mathrm{0}, 235)$.

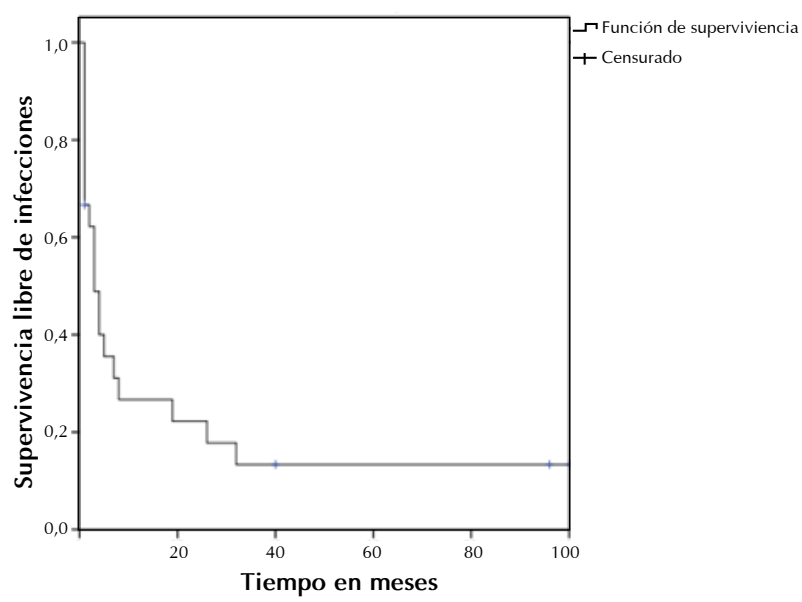

Figura 1. Tiempo libre de infección urinaria del injerto renal con ureterostomía cutánea

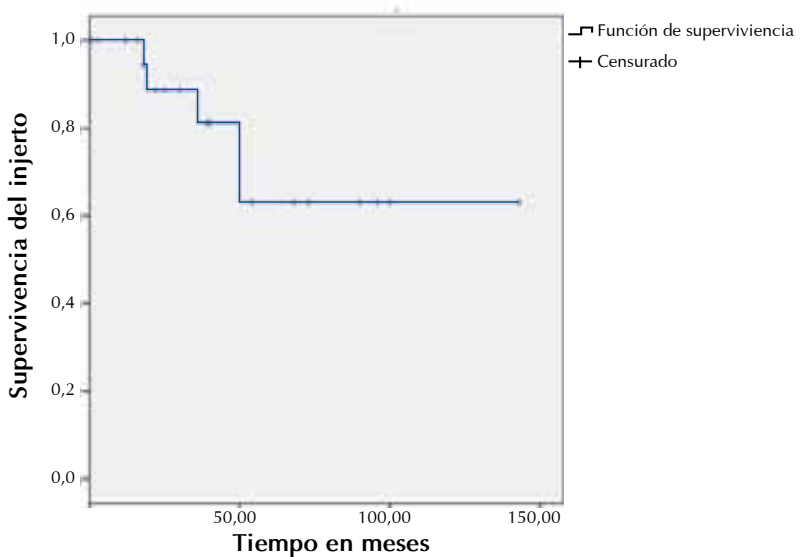

Figura 2. Supervivencia de injerto renal con ureterostomía cutánea



Figura 3. Supervivencia del paciente con trasplante renal con ureterostomía cutánea

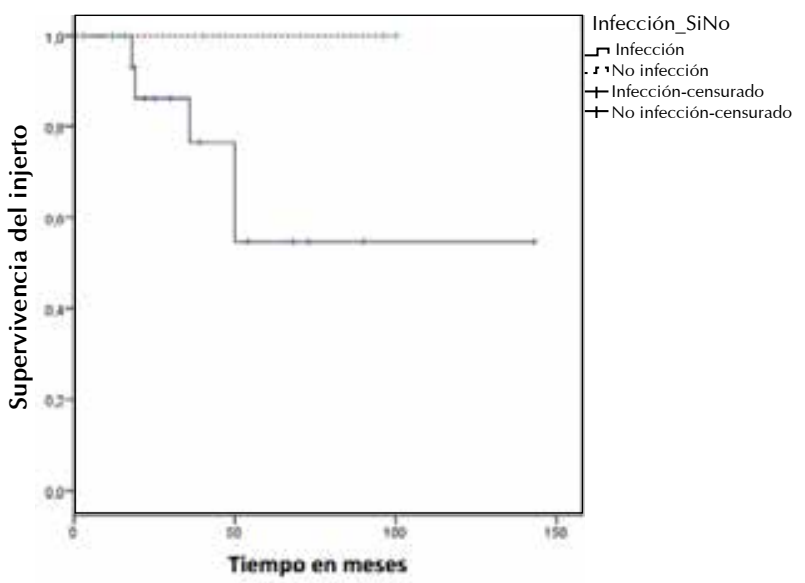

Figura 4. Comparación de la supervivencia del injerto con ureterostomía cutánea e infección urinaria con aquella en casos sin infección urinaria 


\section{Discusión}

A pesar de que el $15 \%$ de los pacientes con trasplantes puede llegar a presentar alguna alteración vesical al momento del trasplante ${ }^{\mathrm{I}}$, no se ha establecido la mejor forma para manejarla. La ureterostomía cutánea es una técnica poco utilizada como alternativa de derivación urinaria en pacientes con trasplante renal. Hasta el momento, en el mundo solo se han reportado 24 pacientes de cuatro diferentes series de $\operatorname{casos}^{12-18}$. En la presente serie, se cuenta con 24 pacientes que correspondieron al o,55 \% de los 4294 pacientes que recibieron trasplantes renales en 39 años estudiados en el Hospital.

Entre las indicaciones para ureterostomía reportadas en las series, la vejiga neurogénica fue la más frecuente ( 13 casos). Otras causas descritas fueron cistitis por tuberculosis ( 4 casos), extrofia vesical, otras alteraciones del seno urogenital y, por último, valvas de la uretra posterior.

A diferencia de lo anterior, en la presente serie, las valvas de la uretra posterior fueron la principal causa de alteración vesical 6/24 (25\%) que requirió ureterostomía en el momento del trasplante renal. Esto se explica porque cerca de $45 \%(\mathrm{II} / 24)$ de los pacientes trasplantados en esta serie eran menores de I8 años y las valvas de la uretra posterior pueden producir disfunción renal hasta en $59 \%$ (I4/24) de los casos pediátricos ${ }^{19}$.

Otras alteraciones uretrales encontradas fueron duplicación uretral, estenosis por trauma pélvico y estenosis por resección transuretral y epispadias. La estenosis uretral solo fue identificada en el intraoperatorio al pasar la sonda vesical durante el trasplante, a pesar del protocolo pretrasplante que no la detectó con anticipación y que obligó al cirujano a practicar la ureterostomía como técnica de derivación. Llama la atención, como en otras series, la pérdida de la vejiga por cistitis tuberculosa, que no permitieron más alternativa que la ureterostomía. En nuestra serie se presentaron dos casos. Esto puede ser explicado por la asociación con tuberculosis intestinal, que no permite para su derivación utilizar otros segmentos intestinales para la reconstrucción como en la ureteroileostomía tipo Bricker ${ }^{20-23}$.
Debido a la alta frecuencia de complicaciones perioperatorias (que alcanzan el 7I,4 \%) y de estenosis en el largo plazo (del I7,4\%) reportada en series con ureterostomía cutánea terminal en casos de cirugía urológica sin trasplante ${ }^{24}$ y la frecuencia de complicaciones $(45 \%)$ de la ureterostomía terminal en las series de trasplante ${ }^{12,13}$ en nuestro centro se decidió a partir del año 20II, realizar la técnica de ureterostomía en asa lateral tipo Amin ${ }^{14,16}$. En 75 \% (18/24) de los casos reportados en esta serie, se practicó la ureterostomía terminal y solo en seis se utilizó la técnica en asa lateral de Amin ${ }^{9-1,1,16}$.

La tasa de falla de la ureterostomía fue del $4 \mathrm{I}, 7 \%$ y su causa más frecuente fue la estenosis que originaba disfunción del injerto. Esto se explica por el mayor número de ureterostomías terminales practicadas durante el periodo de estudio y su mayor tiempo de seguimiento, y se correlaciona apropiadamente con las experiencias reportadas por Prieto, et al. ${ }^{12}$, y Purohit, et al. ${ }^{13}$. A pesar de lo anterior, la mayoría de los casos requirieron procedimientos relativamente simples (dilatación y reimplantes) para recuperar la funcionalidad del injerto en su totalidad. Solo un caso terminó en pérdida completa de las vías urinarias.

La frecuencia de infecciones urinarias en los trasplantados con ureterostomía se encuentra entre el 33 y el $83 \%$, ninguna asociada con pérdida del injerto o muerte del paciente ${ }^{12-16}$. Prieto, et al., reportaron que la tasa anual de infecciones fue de 3,I en los pacientes trasplantados con ureterostomía ${ }^{12}$.

Las tasas de infección urinaria varían con las diferentes opciones terapéuticas. Con la cistoplastia de aumento, las tasas de infección urinaria se encuentran alrededor del $56 \%$ y cerca del $32 \%$ de los casos requieren hospitalización ${ }^{7,20-22}$. La derivación ileal se acompaña de infección urinaria en el $25 \%$ de los pacientes ${ }^{4,23,25}$. Sobre otras alternativas, como las derivaciones urinarias continentes, solo se encuentran reportes de caso y, con tan altas frecuencias de infección, que informan la necesidad de profilaxis con antibióticos ${ }^{4,5}$. 
En la presente serie, el $83,3 \%{ }^{20 / 24}$ de los pacientes presentaron al menos un episodio de infección urinaria, y el 50 \% (I2/24) tuvo algún episodio de infección en el primer año de seguimiento. Esto puede explicarse por la alta tasa de estenosis de las ureterostomías (33,3\%; 8/24), la exposición de la orina a la flora microbiana cutánea y al reflujo ureteropiélico generado por la estenosis ${ }^{26}$. De hecho, es posible que la técnica de asa lateral de Amin pudiera impactar en la frecuencia de estenosis. Sin embargo, la presencia de estenosis no explica todas las infecciones urinarias.

A pesar de la gran frecuencia de la infección urinaria, hubo pérdida del injerto solo en el 20,8\% (5/24) y solo uno de los cinco casos se explicaba por pérdida de las vías urinarias y las infecciones a repetición. Esto obliga a evaluar otros factores asociados con la pérdida del injerto en futuros análisis.

Por otro lado, al comparar los injertos que estuvieron infectados con los que no se infectaron, se encontró una importante disminución de la supervivencia de los primeros (IOO \% Vs. $54 \%$ ) a los 50 meses de seguimiento. Esto no se había evidenciado en otras series debido a las muestras tan pequeñas y a los periodos de seguimiento tan variables. No obstante, sugiere la necesidad de mantener con profilaxis antibiótica a este tipo de pacientes.

En pacientes con ureterostomía, la supervivencia de los injertos renales se encuentra entre el 83 y el Ioo \% después de tres años de seguimiento, sin mortalidad perioperatoria y con supervivencia de los pacientes cercana al 90,I $\%$, con periodos de seguimiento que alcanzaron hasta los I8 años en promedio ${ }^{12-16}$. De acuerdo con la Organ Procurement and Transplantation Network (OPTN), en general, la supervivencia de los injertos cadavéricos es del $77 \%$ (versus $87,9 \%$ en injerto vivo relacionado) a los tres años y del $66,8 \%$ (versus $79,8 \%$ en injerto vivo relacionado) a los cinco años; además, la supervivencia de los pacientes es de $88,2 \%$ y $88,1 \%$, respectivamente ${ }^{27}$. Estos tiempos son comparables con los de la presente serie, en la cual la supervivencia del injerto fue de $85,4 \%$ a los tres años de seguimiento y, la de los pacientes, cercana al 90,2 \% después de un seguimiento de Io,8 años en promedio.

\section{Conclusión}

La ureterostomía cutánea es una alternativa segura para la derivación urinaria de pacientes trasplantados renales con alteraciones de la vejiga que no permite su uso o preparación antes del trasplante. Sin embargo, la gran frecuencia de infecciones urinarias asociadas impacta la supervivencia del injerto.

\section{Cumplimiento de normas éticas}

Consentimiento informado. Este estudio es una revisión retrospectiva de historias clínicas y, como tal, no hay necesidad de un consentimiento informado. El Comité de Ética Institucional aprobó el diseño y la metodología del estudio.

Declaración de conflicto de intereses. Los autores declaramos que no hay conflicto de intereses ni plagios.

Fuentes de financiación: recursos propios de los autores.

\section{Referencias}

I. Cerilli J, Anderson GW, Evans WE, Smith JP. Renal transplantation in patients with urinary tract abnormalities. Surgery. 1976;79:248-52.

2. Crowe A, Cairns HS, Wood S, Rudge CJ, Woodhouse CR, Neild GH. Renal transplantation following renal failure due to urological disorders. NDT Plus. I998;13:2065-9. https://doi.org/IO.IO93/ndt/I3.8.2065

3. Reinberg Y, González R, Fryd D, Mauer SM, Najarian JS. The outcome of renal transplantation in children with posterior urethral valves. J Urol. I988;I4O:I49I-3. https://doi.org/IO.IOI6/Soo22-5347(I7)42082-9

4. Sullivan ME, Reynard JM, Cranston DW. Renal transplantation into the abnormal lower urinary tract. BJU Int. 2003;92:510-5.

https://doi.org/IO.IO46/j.I464-4IOX.2003.04377.X

5. Mishra SK, Muthu V, Rajapurkar MM, Desai MR. Kidney transplantation in abnormal bladder. BJU Int. 2007;23:299-304. https://doi.org/I0.4IO3/0970-I59I.33728

6. Barry JM. Kidney transplantation into patients with abnormal bladders. Transplantation. 2004;77:II20-3. https://doi.org/Io.I097/or.TP.ooooII67II.59454.FI 
7. Nahas WC, Mazzucchi E, Arap MA, Antonopoulos IM, Neto ED, Ianhez LE, et al. Augmentation cystoplasty in renal transplantation: A good and safe option--experience with 25 cases. Urology. 2002;60:770-4. https://doi.org/IO.IOI6/Soo9o-4295(02)01947-7

8. Neild GH, Dakmish A, Wood S, Nauth-Misir R, Woodhouse CR. Renal transplantation in adults with abnormal bladders. Transplantation. 2004;77:I123-7. https://doi.org/IO.IO97/oI.TP.ooooII6712.56265.78

9. Amin M. Terminal loop cutaneous ureterostomy in renal transplantation. Urology. 1992;40:580. https://doi.org/IO.IoI6/0090-4295(92)90422-S

Io. Amin M, Clark R, Howerton LW, Lich R, Jr. Terminal loop cutaneous ureterostomy: An experimental study and its clinical application. J Urol. 1977;II8:383-5. https://doi.org/IO.IOI6/Soo22-5347(I7)58029-5

II. Amin M, Howerton LW, Lich R, Jr. Terminal loop cutaneous ureterostomy: A method of urinary drainage in kidney transplantation. J Urol. 1977;II8:379-82. https://doi.org/IO.IOI6/Soo22-5347(I7)58028-3

I2. Prieto $M$, Sierra $M$, de Francisco AL, Zubimendi JA, Montesinos MG, de la Torriente J, et al. Longterm outcome in renal transplantation with terminal cutaneous ureterostomy. Br J Urol. 1993;72:844-7. https://doi.org/IO.IIII/j.I464-4IoX.I993.tbi6284.X

I3. Purohit RS, Bretan PN, Jr. Successful long-term outcome using existing native cutaneous ureterostomy for renal transplant drainage. J Urol. 2000;163:446-9. https://doi.org/IO.IOI6/Soo22-5347(05)67897-4

I4. Tsai SY, Chang CY, Piercey K, Kapoor A. Terminal loop cutaneous ureterostomy in renal transplantation: An underutilized urinary diversion technique. J Urol. 2005;174:1906-9.

https://doi.org/Io.I097/oI.ju.0ooor76749.86199.I4

I5. Tsai SY, Wong CC, Smith EK, Diciommo P, Ludwin D. Terminal loop cutaneous ureterostomy in renal transplantation. Urology. 1992;40:280-2. https://doi.org/IO.IOI6/0090-4295(92)90493-G

I6. Garrison RN, Bentley FR, Amin M. Terminal loop cutaneous ureterostomy in cadaveric kidney transplantation. Arch Surg. 1989;124:467-9. https://doi.org/IO.IOoI/archsurg.I989.0I4IO0400770I8

I7. McDonald MW, Zincke H, Engen DE, Sterioff S. Adaptation of existing cutaneous ureterostomy for urinary drainage after renal transplantation. J Urol. 1985;133:1026-8.

https://doi.org/IO.IOI6/Soo22-5347(I7)49363-3
I8. Bretan PN, Jr, Purohit RS. Successful long-term outcome utilizing existing native cutaneous ureterostomy for renal transplant drainage without ipsilateral native nephrectomy. Transplantation Proceedings. 2000;32:77I-2. https://doi.org/IO.IOI6/So04I-I345(oo)0o977-5

I9. Dinneen MD, Duffy PG, Barratt TM, Ransley PG. Persistent polyuria after posterior urethral valves. $\mathrm{Br} \mathrm{J} \mathrm{Med}$ Surg Urol. 1995;75:236-40. https://doi.org/IO.IIII/j.I464-4IOX.I995.tbo73I8.x

20. MacGregor P, Novick AC, Cunningham R, Streem S, Kay R, Steinmuller D, et al. Renal transplantation in end stage renal disease patients with existing urinary diversion. J Urol. 1986;135:686-8. https://doi.org/IO.IOI6/Soo22-5347(I7)458I9-8

2I. Hatch DA, Koyle MA, Baskin LS, Zaontz MR, Burns MW, Tarry WF, et al. Kidney transplantation in children with urinary diversion or bladder augmentation. J Urol. 200I;165:2265-8.

https://doi.org/Io.IOI6/Soo22-5347(05)66I8I-2

22. Hatch DA. Kidney transplantation in patients with an abnormal lower urinary tract. Urol Clin North Am. I994;2I:3II-20.

23. Coosemans W, Baert L, Kuypers D, Maes B, Messiaen $\mathrm{T}$, Vanrenterghem Y, et al. Renal transplantation onto abnormal urinary tract: Ileal conduit urinary diversion. Transplantation Proceedings. 200I;33:2493-4. https://doi.org/IO.IOI6/Soo4I-I345(OI)O2O74-7

24. Martínez-Pineiro L, Julve E, García-Cardoso JV, Madrid J, de la Peña? J, Martínez-Pineiro JA. Review of complications of urinary diversions performed during a 6-year period in the era of orthotopic neobladders. Arch Esp Urol. 1997;50:433-45.

25. Rischmann P, Malavaud B, Bitker MO, Chretien Y, Dawahara M, Descottes JL, et al. Results of 5I renal transplants with the use of bowel conduits in patients with impaired bladder function: A retrospective multicenter study. Transplantation Proceedings. 1995;27:2427-9.

26. Martin DC, Mims MM, Kaufman JJ, Goodwin WE. The ureter in renal transplantation. J Urol. I969;IOI:680-7. https://doi.org/IO.IOI6/Soo22-5347(I7)624OI-7

27. U.S. Department of Health and Human Services. Organ Procurement and Transplantation Network (OPTN). Kidney Kaplan-Meier graft survival rates for transplants performed: 1997-2004 based on OPTN data as of May 3I, 20I3. Fecha de consulta: 3 de octubre de 2019. Disponible en: http://optn.transplant.hrsa.gov/latestData/rptStrat.asp 